\title{
Image Findings Following Vertebroplasty in Osteoporotic Vertebral Compression Fractures: Bone Healing and Sagittal Alignment
}

\author{
Hirotaka Ikeda $^{1 *}$, Misako Nishio ${ }^{1}$, Shin Matsuoka ${ }^{1}$, Brandon D. Lohman ${ }^{1}$, \\ Shoichiro Matsushita ${ }^{1}$, Yukihisa Ogawa ${ }^{1}$, Shingo Hamaguchi ${ }^{1}$, Yasuo Nakajima ${ }^{1}$, \\ Atsushi Kojima², Yoshiaki Torii ${ }^{2}$, Yutaka Sasao \\ ${ }^{1}$ Department of Radiology, St. Marianna University School of Medicine, Kawasaki, Japan \\ ${ }^{2}$ Department of Orthopedic Surgery, St. Marianna University School of Medicine, Kawasaki, Japan \\ Email: ${ }^{*}$ ikeda-gif@umin.net
}

Received June 11, 2013; revised July 11, 2013; accepted July 18, 2013

Copyright (C) 2013 Hirotaka Ikeda et al. This is an open access article distributed under the Creative Commons Attribution License, which permits unrestricted use, distribution, and reproduction in any medium, provided the original work is properly cited.

\begin{abstract}
Purpose: To clarify the effect of percutaneous vertebroplasty for vertebral compression fracture by assessing the changes of radiographic and CT image findings. Materials and Methods: A retrospective radiological analysis comprising 101 vertebrae of 48 patients who underwent percutaneous vertebroplasty for painful osteoporotic vertebral compression fracture was conducted. Whole spine radiographs and CT images were compared in patients preoperatively and 6 months postoperativey. Sagittal Cobb angles in three regions, sagittal vertical axis, and pelvic tilt were measured using whole spine lateral radiographs. CT findings due to the vertebral compression fracture, its healing process, and complications were evaluated. Results: On radiographs, sagittal alignment had an average gain of no more than $2.5^{\circ}$ in each region. Sagittal vertical axis and pelvic tilt did not show significant change. Out of 68 vertebrae that demonstrated cortical disruption on preoperative CT, 37 (54\%) demonstrated fusion of disrupted cortex on postoperative CT. Conclusion: No significant difference was observed between preoperative and postoperative spinal sagittal alignment on radiographs. However, CT did reveal healing process through fusion of disrupted cortex, intervertebral bridging, and increased density of cancellous bone. It is suggested that cement in the space of fracture may play a role in both mechanical stability and bone union.
\end{abstract}

Keywords: Vertebroplasty; Polymethylmetacrylate; CT; Sagittal Alignment; Bone Union

\section{Introduction}

Osteoporotic vertebral compression fracture (VCF) is a common cause of back pain in the elderly population and is known to be associated with neurologic compromise, permanent disability and spinal deformity [1]. In addition to these commonly associated ailments, VCF may also progress into spinal kyphosis which in fact, may alter spinal biomechanics consequently affecting spinal erector muscles and therefore causing severe fatigue and pain. It may also involve various bodily systems and cause respiratory dysfunction, gastro-esophageal reflux disease, loss of appetite and decrease both lung capacity and abdominal space [2].

To this end, percutaneous vertebroplasty (PVP) appears to be the treatment of choice where conservative

\footnotetext{
"Corresponding author.
}

management has failed [3,4]. PVP is a minimally invasive procedure that effectively addresses severe back pain associated with VCF and helps prevent kyphosis progression. Theoretically, restoring the overall spinal sagittal alignments in VCF patients is believed to hold obvious benefits. However, although several authors reported that PVP effectively reduced local kyphosis in fractured vertebrae $[5,6]$, few have yet reported PVP's role in improving overall sagittal alignment. Additionally, multi-detector computed tomography (MDCT) effectively evaluates any morphological changes and overall fracture healing process $[7,8]$.

To our knowledge, no report has yet analyzed postPVP osteoporotic VCF healing process from CT imaging and the purpose of this present study was to assess overall spinal alignment changes as well as examine VCF bony healing process based on pre and post-PVP MDCT. 


\section{Materials and Methods}

\subsection{Patients}

Over a three-year period (February 2009 to March 2011) our institution retrospectively assessed 162 post-PVP cases for osteoporotic VCF. The inclusion criteria were preand 6 months post-PVP interventions with both spine CT in the supine position and whole spine lateral radiographs in the standing position. Exclusion criteria consisted of additional PVP treatment or surgery within 6 months after PVP $(n=4)$, as well as imaging artifacts $(n=1)$. Out of the 162 patients who underwent PVP, 53 met the inclusion criteria, 5 were excluded, and 48 with a total number of 101 vertebral compression fractures were enrolled to this study. Baseline characteristics of the patients are shown in Table 1. Our institutional review board approved this study and waived the need for informed consent.

\subsection{PVP Procedures}

The PVP procedures were performed by 4 interventional radiologists (K.T., S.H., Y.O., M.N.) with at least 4 years of experience, using a single $\mathrm{C}$-arm fluoroscopy and consisted of transpedicular bone cement injection under the isocenter puncture method (ISOP) [9]. In the fluoroscopy suite patients underwent sedation with intravenous administration of $25 \mathrm{mg}$ hydroxyzine hydrochloride and $15 \mathrm{mg}$ pentazocine in which subsequently a 23gauge needle with $10 \mathrm{~mL} \mathrm{1 \%} \mathrm{Lidocaine} \mathrm{was} \mathrm{inserted} \mathrm{in}$ the vicinity of the targeted vertebra. A 13- or 15-gauge bone biopsy needle was placed transpedicularly in the anterior two thirds of the fractured vertebral body. Barium-opacified polymethylmethacrylate (PMMA) bone cement was injected under continuous fluoroscopic imaging. The injection was terminated when the cement spread to the posterior quarter of the vertebra or cement leakage occurred. The needle was removed and the patients rested in the supine position for at least 2 hours.

Table 1. Characteristics of patients.

\begin{tabular}{|c|c|}
\hline Characteristics & Value \\
\hline Age (year old) & $76 \pm 6$ \\
\hline Sex (Female/Male) & $35 / 13$ \\
\hline Height $(\mathrm{cm})$ & $145.6 \pm 19.6$ \\
\hline Body weight (kg) & $49.0 \pm 7.9$ \\
\hline Body Mass Index $\left(\mathrm{kg} / \mathrm{cm}^{2}\right)$ & $22.2 \pm 4.1$ \\
\hline $\begin{array}{l}\text { History of Parkinson's disease } \\
\text { (number of cases) }\end{array}$ & 4 \\
\hline $\begin{array}{l}\text { History of cerebral vascular accident } \\
\text { (number of cases) }\end{array}$ & 0 \\
\hline $\begin{array}{l}\text { History of rheumatoid arthritis } \\
\text { (number of cases) }\end{array}$ & 0 \\
\hline $\begin{array}{l}\text { steroid use more than } 5 \mathrm{mg} / \text { day } \\
\text { (number of cases) }\end{array}$ & 4 \\
\hline
\end{tabular}

Indications for PVP were as follows: 1) older than 60 years of age; 2 ) continuous pain lasting for a period of over 6 weeks despite conservative therapy with bed rest, physical therapy, and analgesics; 3) bone edema or pseudoarthrosis at fractured vertebrae on CT or MRI; and 4) VCF at T5 or lower. The exclusion criteria were as follows: 1) infection; 2) coagulopathy; 3) severe cardiopulmonary comorbidity; 4) suspected underlying malignancy; 5) destroyed posterior wall with neurological symptoms; and 6) severe biconcave type fracture formed consent.

\subsection{Imaging Analysis}

\subsubsection{Whole Spine Radiographs \& Spinal CT}

Lateral whole spine radiographs in the standing position were obtained both pre- and six months post-PVP procedures. Two diagnostic radiologists (H.I. and S.M.) then performed imaging analysis using imaging viewer (Yokogawa Electronic Corporation, Japan) and compared sagittal tilt angle of 3 separate regions following the Cobb method; (thoracic kyphosis (T5-T12), lumbar lordosis (T12-S1) and thoracolumbar kyphosis (T10-L2)) as well as sagittal vertical axis (SVA) and pelvic tilt (PT) $[10,11]$ (Figure 1).

All patients were scanned with a 64-MDCT scanner (Aquilion 64, Toshiba Medical Solutions). The scanning parameters were as follows: $120 \mathrm{kVp}$; a $0.5 \mathrm{~mm}$ collimation; a 0.75 -second rotation time; a pitch of 0.64 ; 3-mm-thick reconstructions; and $3-\mathrm{mm}$ reconstruction increments with a bone reconstruction algorithm (FC31). Sagittal and coronal images were reconstructed and compared from the data sets obtained pre- and post-PVP with MDCT using a ZIO workstation (ZIOSOFT, Japan).

\subsubsection{Semiquantitative Method}

Vertebral fractures were assessed following the semiquantitative method which was originally established by Genant et al. [12] and consists of a visual evaluation of collapsed vertebrae. This method is documented to have higher inter- and intra-observer agreement when compared to the more widely utilized quantitative method [12]. Grade I represents deformity with vertebral height reduction ranging from $20 \%$ to $25 \%$, Grade II reduction from $25 \%$ to $40 \%$ and Grade III reduction $>40 \%$. The semiquantitative method can also grade vertebral fracture from MDCT remodeled sagittal views and can be as useful as a traditional lateral radiographs $[13,14]$.

\subsubsection{CT Imaging}

CT imaging of the pre- and post-PVP procedures can clearly delineate the healing processes involved within and around the fracture zone of the vertebral body. For this purpose we divided the findings according to: 1) findings from fracture, 2) findings from healing process 

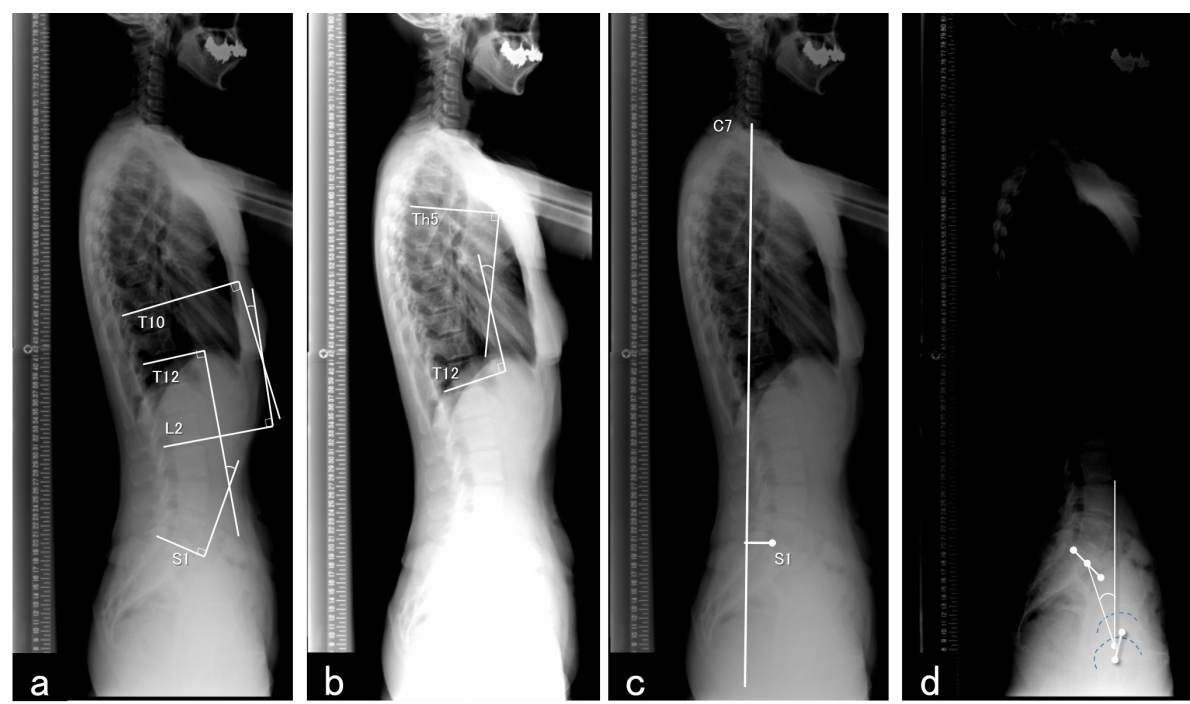

Figure 1. 50-year-old healthy woman with whole spine radiograph that shows the ways of measurements. (a) Thoracolumbar (T10-L2) and lumbar (T12-S1) lordotic angle measurement lines using Cobb method are shown. (b) Thoracic kyphotic angle (T5-T12) measurement lines using Cobb method are shown. (c) Sagittal balance that is the distance from posterior edge of sacral endplate to $C 7$ vertical axis is measured. (d) Pelvic tilt angle is defined as the angle between a vertical line and a line connecting the midpoint of bilateral femoral heads and the midpoint of sacral endplate.

and 3) complications.

1. Findings from fracture are characterized with 1) the minimal spinal canal diameter at the fractured level on sagittal reconstructed CT data, inspecting how much of the spur or PMMA protruded into spinal canal (Figure 2); 2) Cortical bone disruption was observed in the superior/inferior endplate, anterior, posterior and/or lateral wall of vertebra. (Figure 3(a)).

2. Findings from healing process describe 1) the fusion of disrupted cortex that can be detected on follow-up $\mathrm{CT}$ and compared with the pre-PVP CT images (Figure 3(b)); 2) Intervertebral bony bridging structures attach the PVP-treated fractured vertebra with the immediately adjacent superior or inferior vertebra with the help of elongated and fused spurs often found in the anterior surface of the vertebrae (Figure 4); 3) Increased cancellous bone density may be representative of cancellous bone stiffness resulting from intravertebral ossification from the union of a fractured vertebral body (Figure 5).

3. Finally, Complications were generally associated with cement leakage from the treated vertebral body (Figure 3(b)) and also additional vertebral fractures that appear on follow-up CT imaging.

\subsection{Statistical Analysis}

The results of imaging analysis of the whole spine radiograph are provided as mean \pm standard deviation of measured angles and length. The results of CT image analysis are provided as the percentage of the vertebrae where each finding was obtained. Statistical analysis was performed using commercially available software (JMP,
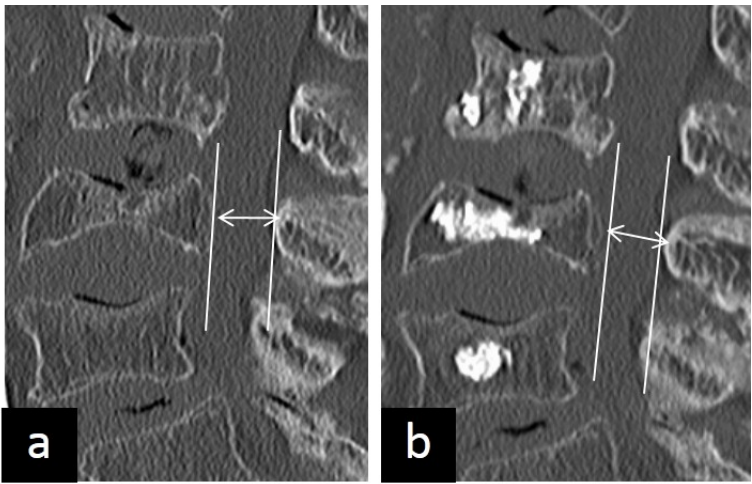

Figure 2. Minimal spinal diameters of sagittal reconstructed images of both preoperative (a) and 6 months postoperative (b) CT were measured.
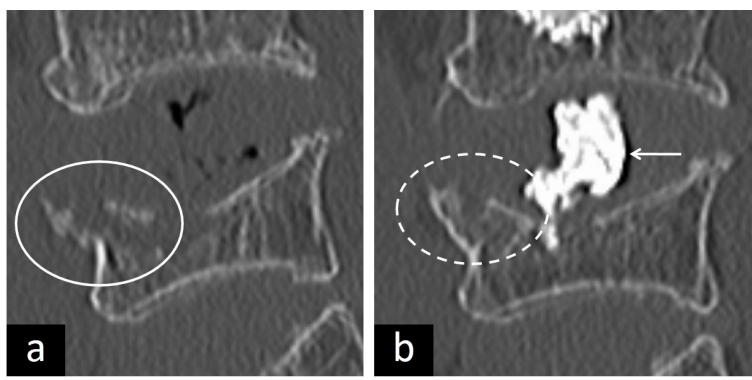

Figure 3. 78-year-old woman with osteoporotic vertebral compression fractures who underwent percutaneous vertebroplasty at T10-T12. (a) Sagittal reconstruction of preoperative spine CT shows disrupted cortex at the fractured vertebra (circle). (b) Fusion of disrupted cortex was seen at the same vertebra on the CT taken 6 months after PVP (dashed circle). Cement leakage from the treated vertebra was also seen in the intervertebral space (arrow). 

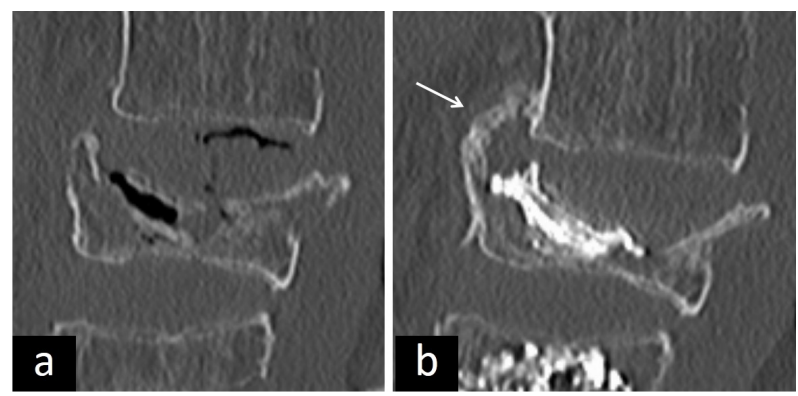

Figure 4. 80-year-old woman with osteoporotic vertebral compression fractures who underwent percutaneous vertebroplasty at T12-L1. (b) Sagittal reconstructed CT image 6 months postoperatively shows anterior bony bridging connecting the T12 vertebrae on which PVP was performed and its adjacent T11 vertebra (arrow). (a) Bony bridging was not seen on the CT taken before the PVP procedure.
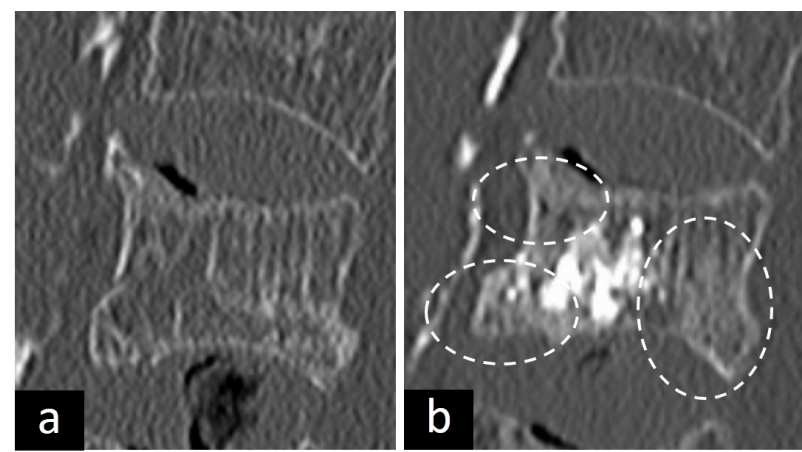

Figure 5. 82-year-old woman with osteoporotic vertebral compression fractures who underwent percutaneous vertebroplasty at L3. (b) Increased cancellous bone density (dashed circles) seen at the structure surrounding the injected PMMA cement on the reconstructed axial image of spine CT taken 6 months postoperatively, compared to CT taken preoperatively (a).

version 8.0.2, SAS Institute). The t-test was applied to determine whether the results of the measurements on whole spine radiographs and the minimal spinal diameter on CT were significantly different pre- and post-PVP. A $p$ value of less than 0.01 was considered to indicate statistical significance.

\section{Results}

The mean age of the patients was $76 \pm 6$ years $(35$ women and $13 \mathrm{men})$. The mean follow-up period was 6.2 \pm 0.7 months ( $5-7$ months). The treated levels were distributed from T7 to L5: 2 in T7, 1 in T8, 2 in T9, 4 in $\mathrm{T} 10,6$ in T11, 21 in T12, 22 in L1, 22 in L2, 11 in L3, 7 in L4, and 3 in L5.

On whole spine radiographs, we found no significant difference $(p<0.01)$ between thoracic, thoracolumbar kyphosis, lumbar lordosis, SVA, and PT angle in preand post-PVP imaging investigations (Table 2).

Out of a total of $101 \mathrm{VCF}$ in 48 patients, 97 vertebrae
Table 2. The result of measurements using lateral whole spine radiograph.

\begin{tabular}{ccccc}
\hline Measurements & Preoperation & Postoperation & post - pre & p value \\
\hline $\begin{array}{c}\text { Thoracic (T5-12) } \\
\text { kyphotic angle }\end{array}$ & $36.4 \pm 17.4$ & $37.4 \pm 18.8$ & $0.9 \pm 8.3$ & 0.611 \\
$\begin{array}{c}\text { Thoracolumbar } \\
\text { (T10-L2) kyphotic } \\
\text { angle }\end{array}$ & $34.5 \pm 16.7$ & $36.9 \pm 17.3$ & $2.5 \pm 7.1$ & 0.013 \\
$\begin{array}{c}\text { Lumbar (T12-S1) } \\
\text { kyphotic angle }\end{array}$ & $-23.6 \pm 18.6$ & $-24.4 \pm 19.0$ & $-0.8 \pm 10.0$ & 0.695 \\
$\begin{array}{c}\text { Sagittal balance } \\
\text { (mm) }\end{array}$ & $78.7 \pm 47.2$ & $82.5 \pm 52.9$ & $3.8 \pm 31.9$ & 0.383 \\
Pelvic tilt angle & $33.8 \pm 11.0$ & $34.2 \pm 11.0$ & $0.4 \pm 5.4$ & 0.473 \\
\hline
\end{tabular}

Positive value of Cobb angle indicate kyphosis, negative values of Cobb angle indicate lordosis. None of the measurements showed statistically significant difference between pre- and 6 months post-PVP.

retained the same type of deformity while 94 vertebrae demonstrated identical degree of severity on both preand post-PVP CT analysis.

Following a semi-quantitative method inspection, we did not identify any significant differences in morphology and severity of fracture on sagittal reconstructed images of CT (Figure 6).

The mean minimum spinal diameter was $11.2 \mathrm{~mm} \pm$ 2.4 on pre-PVP CT and $10.9 \mathrm{~mm} \pm 2.6$ on post-PVP CT, which did not show any significant difference $(p=0.03)$.

The mean preoperative and postoperative loss of spinal canal diameter was $0.3 \mathrm{~mm} \pm 1.4$ (Table 3).

Out of 101 vertebrae, 68 vertebrae $(68 \%)$ showed cortical disruption on Pre-PVP CT in which 37 vertebrae (54\%) demonstrated fusion of disrupted cortex on PostPVP CT.

Furthermore, of the 101 vertebrae, 26 (26\%) were identified with increased cancellous bone density and 11 (11\%) demonstrated intervertebral bridging. However 47 vertebrae $(47 \%)$ displayed PMMA leakage while new fractures were identified in $18(38 \%)$ of 48 cases (Table 4).

\section{Discussion}

To our knowledge, this is the first report describing postPVP healing process of vertebral compression fractures with CT imaging as well as its accompanying spinal stabilization.

As a matter of fact, Post-PVP CT imaging demonstrated clear healing process findings containing cortical fusion, intervertebral bridging formation and increased cancellous bone density. However, no significant differences between morphological changes, severity of vertebral fracture could be obtained from pre- and post-PVP CT imaging analysis. From these evaluations, PVP proved to provide effective internal fixation to the fractured vertebrae and maintain spinal alignment stabilization hence resulting in pain reduction. Albeit the lack of 


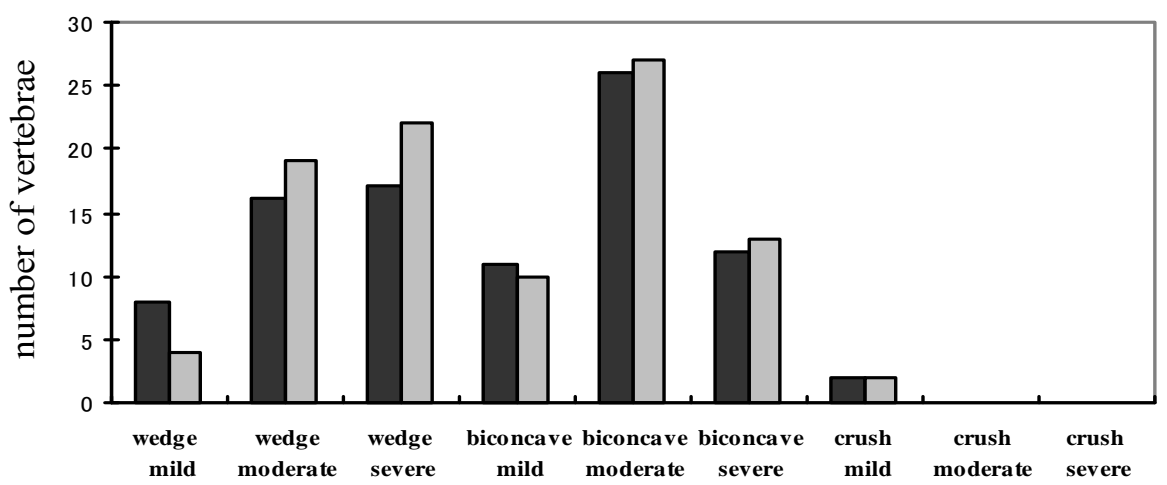

Figure 6. Distribution patterns of morphology and severity of vertebral compression fracture regarding the Semiquantitative method.

Table 3. CT findings of vertebral compression fracture and its number of vertebrae $(\%)$.

\begin{tabular}{ccccc}
\hline \multirow{2}{*}{ CT findings } & Preoperation & \multicolumn{3}{c}{ Postoperation } \\
\cline { 2 - 5 } & Positive & Better & Worse & No change \\
\hline $\begin{array}{c}\text { Cortical } \\
\text { disruption }\end{array}$ & $68(67.3)$ & $37(36.6)$ & $18(17.8)$ & $46(45.5)$ \\
$\begin{array}{c}\text { Hyperdense } \\
\text { cancellous bone } \\
\text { Minimal spinal } \\
\text { diameter }(\mathrm{mm})\end{array}$ & $68(67.3)$ & $26(25.7)$ & $18(17.8)$ & $59(58.4)$ \\
\hline
\end{tabular}

Table 4. Postoperative CT findings and its number of vertebrae (\%).

\begin{tabular}{cc}
\hline Fingings of healing process & \\
\hline Fusion of disrupted cortex & $37(36.6)$ \\
Intervertebral bridging & $11(10.9)$ \\
Increased density of cancellous bone & $26(25.7)$ \\
\hline Findings of complications & \\
\hline Cement leakage & $47(46.5)$ \\
New fracture & $18(37.5)$ \\
\hline
\end{tabular}

significant improvement in Cobb angle [thoracic (T5-T12), thoracolumbar (T10-L2) and lumbar (T12-S1)], sagittal vertebral axis (spinal balance parameter) and pelvic tilt (spinopelvic parameter) (Table 2); it is noteworthy to mention that among all CT studies, cortical fusion was most commonly observed and represented complete union of fractured vertebrae after PVP (Figure 3(b)).

The PVP cementing action creates an intervertebral bridging consisting of elongated spur formations that appear to enhance the biomechanical stability of the vertebral unit including not only the treated vertebra in question but also the adjacent vertebrae (Figure 4(b)). Furthermore, CT studies of PVP-treated vertebrae displayed marked increased density within cancellous bone, most likely resulting from bony tissue reaction to PMMA which thereby, precipitate into a callus formation that would lead to the vertebral fracture fixation (Figure 5).

In concordance with Braunstein et al. whom reported microscopic callus formations within woven bone surrounding the injected PMMA in human osteoporotic VCF [15], we likewise detected post-PVP hyperdensities in cancellous bones in 26 vertebrae representing fracture healing. However, hyperdense cancellous formations were also detected in 68 pre-PVP vertebrae and may represent normal healing process occurring within the zones of fracture. Although it has not been elucidated yet whether PMMA actually fuses with human vertebrae [15-18], it appears to play a role as an inducing agent in the normal process of fracture healing. Studies comparing the histomorphometry and CT findings of PMMA injected vertebrae with non-PMMA injected vertebrae are warranted in order to clarify and confirm with certainty this bony reaction.

In addition, we evaluated the minimal spinal diameter within the sagittal spinal canal in order to assess the risk of neural complication from PVP. Indeed, intra-vertebral cement injection during PVP procedure has been reported to cause spinal canal narrowing, thereof forming protruding bony fragments within the spinal canal which potentially may increase the risk of neurological deficits $[19,20]$. The authors of this study did not identify any significant changes in minimal spinal diameter on both pre- and post-PVP and assumed that the spinal canal narrowing held a low risk of neural complications (Figure 2).

In comparison to previous reports, contrarily to our expectations, this study exhibited, a noticeably high number of newly detected vertebral fractures upon follow-up examinations. We attributed this prevalence to the fact that the newly diagnosed VCFs were detected with CT imaging in contradistinction with recent reports which identified post-PVP fractures utilizing radiographic 
images [21,22]. CT imaging identifies with higher sensitivity and more accuracy the occurrence of newly developed VCF that would not be detected otherwise [14]. PVP is recognized to improve local kyphosis [5,6] however our study indicated no significant improvement in overall kyphosis or sagittal balance. Actually, it appears that the results of this present study rather demonstrated that progression of kyphosis may have possibly increased slightly during the follow-up period, presumably as kyphosis, when left untreated, would follow its natural course and aggravate.

Admittedly, kyphosis correction improves quality of life and SVA is a critical element known to improving health-related quality of life [23]. However our data did not show any improvement of SVA values. It has been reported that local kyphotic angle tend to worsen within 2 years after deformity reduction [24,25]. It is therefore our contention that PVP may hold value only in correcting local kyphosis by decreasing fracture mobility but may not decidedly contribute to the overall sagittal alignment and PVP's role may limit itself to the fixation of vertebral body fractures.

After examining pre-and post-PVP stability of overall sagittal alignment and vertebral fracture union our results indicated that mechanical stabilization of the spine by internal fixation of fractured vertebrae appeared to be the dominant factor in pain reduction in post-PVP procedures.

Detailed analysis of the fracture healing process is often difficult to detect on MRI or spine radiographs, and more so during the early stage of the bone healing process, at a time when a definite evaluation of the implemented PVP is crucial to determine subsequent management procedures. To this effect, follow-up CT imaging can be a viable alternative although the risk of radiation associated with CT deserves judicious planning and caution.

Given the retrospective nature of this study, we acknowledge that there are a number of inherent limitations, including the small sample size, lack of a control group as well as pathological investigation of bone healing. We initially aimed to define which imaging data pertained most relevantly to pre- and post-PVP in order to set the ground for future studies that would correlate such investigation with clinical outcomes. In fact, a longer follow-up period and a clinical assessment addressing the degree of pain reduction and quality of life improvement could provide valuable insight regarding PVP treatment during VCF. A prospective analysis confirming these findings in future studies is warranted

\section{Conclusion}

In conclusion, percutaneous vertebroplasty in case of vertebral compression fracture does not significantly im- prove overall spinal sagittal alignment as previously reported with local kyphotic angle in radiographic imaging. However, CT investigations determined the presence of cortical fusion, inter-vertebral bridging formation and increased cancellous bone density. PVP appears to be involved in both mechanical stability and bone union of vertebral compression fractures.

\section{Acknowledgements}

The authors are grateful to Dr. Kenji Takizawa who supervised this project.

\section{REFERENCES}

[1] J. J. Verlaan, F. C. Oner and W. J. Dhert, "Anterior Spinal Column Augmentation with Injectable Bone Cements," Biomaterials, Vol. 27, No. 3, 2006, pp. 290-301. doi:10.1016/j.biomaterials.2005.07.028

[2] S. R. Garfin, H. A. Yuan and M. A. Reiley, "New Technologies in Spine: Kyphoplasty and Vertebroplasty for the Treatment of Painful Osteoporotic Compression Fractures," Spine (Phila Pa 1976), Vol. 26, No. 14, 2001, pp. 1511-1515.

[3] M. O. Baerlocher, P. L. Munk, D. M. Liu, G. Tomlinson, M. Badii, et al. "Clinical Utility of Vertebroplasty: Need for Better Evidence," Radiology, Vol. 255, No. 3, 2010, pp. 669-674. doi:10.1148/radiol.10092107

[4] W. T. Ploeg, A. G. Veldhuizen, B. The and M. S. Sietsma "Percutaneous Vertebroplasty as a Treatment for Osteoporotic Vertebral Compression Fractures: A Systematic Review," European Spine Journal, Vol. 15, No. 12, 2006, pp. 1749-1758. doi:10.1007/s00586-006-0159-Z

[5] M. Krauss, H. Hirschfelder, B. Tomandl, G. Lichti and I. Bar, "Kyphosis Reduction and the Rate of Cement Leaks after Vertebroplasty of Intravertebral Clefts," European Radiology, Vol. 16, No. 5, 2006, pp. 1015-1021. doi:10.1007/s00330-005-0056-6

[6] M. B. Pitton, U. Koch, P. Dreesand and C. Duber, "Midterm Follow-Up of Vertebral Geometry and Remodeling of the Vertebral Bidisk Unit (VDU) after Percutaneous Vertebroplasty of Osteoporotic Vertebral Fractures," CardioVascular and Interventional Radiology, Vol. 32, No. 5, 2009, pp. 1004-1010. doi:10.1007/s00270-009-9521-y

[7] C. R. Krestan, H. Noske, V. Vasilevska, M. Weber, G. Schueller, et al., "MDCT versus Digital Radiography in the Evaluation of Bone Healing in Orthopedic Patients," American Journal of Roentgenology, Vol. 186, No. 6, 2006, pp. 1754-1760. doi:10.2214/AJR.05.0478

[8] J. E. Kuhlman, E. K. Fishman, D. Magid, W. W. Scott Jr., A. F. Brooker and S. S. Siegelman, "Fracture Nonunion: CT Assessment with Multiplanar Reconstruction," Radiology, Vol. 167, No. 2, 1988, pp. 483-488.

[9] S. Sakaino, K. Takizawa, M. Yoshimatsu, Y. Ogawa, K. Yagihashi and Y. Nakajima, "Percutaneous Vertebroplasty Performed by the Isocenter Puncture Method," $R a$ diation Medicine, Vol. 26, No. 2, 2008, pp. 70-75. doi:10.1007/s11604-007-0197-4 
[10] M. F. O’Brien, T. R. Kuklo, K. M. Blanke, et al., "Radiographic Measurement Manual," Spinal Deformity Study Group (SDSG), Medtronic SofamorDanek, 2004.

[11] P. Roussouly and C. Nnadi, "Sagittal Plane Deformity: An Overview of Interpretation and Management," European Spine Journal, Vol. 19, No. 11, 2010, pp. 18241836. doi:10.1007/s00586-010-1476-9

[12] H. K. Genant, C. Y. Wu, C. van Kuijk and M. C. Nevitt, "Vertebral Fracture Assessment Using a Semiquantitative Technique," Journal of Bone and Mineral Research, Vol. 8, No. 9, 1993, pp. 1137-1148. doi:10.1002/jbmr.5650080915

[13] D. Muller, J. S. Bauer, M. Zeile, E. J. Rummeny and T. M. Link, "Significance of Sagittal Reformations in Routine Thoracic and Abdominal Multislice CT Studies for Detecting Osteoporotic Fractures and Other Spine Abnormalities," European Radiology, Vol. 18, No. 8, 2008, pp. 1696-1702. doi:10.1007/s00330-008-0920-2

[14] J. S. Bauer, D. Muller, A. Ambekar, M. Dobritz, M. Matsuura, et al., "Detection of Osteoporotic Vertebral Fractures Using Multidetector CT," Osteoporosis International, Vol. 17, No. 4, 2006, pp. 608-615. doi:10.1007/s00198-005-0023-8

[15] V. Braunstein, C. M. Sprecher, A. Gisep, L. Benneker, K. Yen, et al., "Long-Term Reaction to Bone Cement in Osteoporotic Bone: New Bone Formation in Vertebral Bodies after Vertebroplasty," Journal of Anatomy, Vol. 212, No. 5, 2008, pp. 697-701. doi:10.1111/j.1469-7580.2008.00883.x

[16] M. Libicher, J. Hillmeier, U. Liegibel, U. Sommer, W. Pyerin, et al., "Osseous Integration of Calcium Phosphate in Osteoporotic Vertebral Fractures after Kyphoplasty: Initial Results from a Clinical and Experimental Pilot Study," Osteoporosis International, Vol. 17, No. 8, 2006, pp. 1208-1215. doi:10.1007/s00198-006-0128-8

[17] I. A. Grafe, M. Baier, G. Noldge, C. Weiss, K. Da Fonseca, et al., "Calcium-Phosphate and Polymethylmethacrylate Cement in Long-Term Outcome after Kyphoplasty of Painful Osteoporotic Vertebral Fractures," Spine (Phila Pa 1976) , Vol. 33, No. 11, 2008, pp. 1284-1290.

[18] E. M. Ooms, J. G. Wolke, M. T. van de Heuvel, B.
Jeschke and J. A. Jansen, "Histological Evaluation of the Bone Response to Calcium Phosphate Cement Implanted in Cortical Bone," Biomaterials, Vol. 24, No. 6, 2003, pp. 989-1000. doi:10.1016/S0142-9612(02)00438-6

[19] M. Hoshino, H. Nakamura, H. Terai, T. Tsujio, M. Nabeta, et al., "Factors Affecting Neurological Deficits and Intractable Back Pain in Patients with Insufficient Bone Union Following Osteoporotic Vertebral Fracture," European Spine Journal, Vol. 18, No. 9, 2009, pp. 1279-1286. doi:10.1007/s00586-009-1041-6

[20] T. Hashimoto, K. Kaneda and K. Abumi, "Relationship between Traumatic Spinal Canal Stenosis and Neurologic Deficits in Thoracolumbar Burst Fractures," Spine (Phila Pa 1976), Vol. 13, No. 11, 1988, pp. 1268-1272.

[21] M. J. Nieuwenhuijse, H. Putter, A. R. van Erkel and P. D. Dijkstra, "New Vertebral Fractures after Percutaneous Vertebroplasty for Painful Osteoporotic Vertebral Compression Fractures: A Clustered Analysis and the Relevance of Intradiskal Cement Leakage," Radiology, Vol. 266, No. 3, 2013, pp. 862-870.

[22] N. Tanigawa, S. Kariya, A. Komemushi, M. Nakatani, R. Yagi, et al., "Percutaneous Vertebroplasty for Osteoporotic Compression Fractures: Long-Term Evaluation of the Technical and Clinical Outcomes," American Journal of Roentgenology, Vol. 196, No. 6, 2011, pp. 1415-1418. doi:10.2214/AJR.10.5586

[23] V. Lafage, F. Schwab, A. Patel, N. Hawkinson and J. P. Farcy, "Pelvic Tilt and Truncal Inclination: Two Key Radiographic Parameters in the Setting of Adults with Spinal Deformity," Spine (Phila Pa 1976), Vol. 34, No. 17, 2009, pp. E599-606.

[24] Y. Sasao, A. Kojima, M. Kaneko, A. Fujii, Y. Torii, et al., "Short-Term Outcome of Percutaneous Vertebroplasty (PVP)," Journal of St. Marianna University, Vol. 1, 2010, pp. 83-91.

[25] D. H. Heo, J. H. Choi, M. K. Kim, H. C. Choi, J. H. Jeong, et al., "Therapeutic Efficacy of Vertebroplasty in Osteoporotic Vertebral Compression Fractures with Avascular Osteonecrosis: A Minimum 2-Year Follow-Up Study," Spine (Phila Pa 1976) , Vol. 37, No. 7, 2012, pp. E423-429. 